Turkish Journal of Mathematics

http://journals.tubitak.gov.tr/math/

Research Article

Turk J Math

(2019) 43: 2549 - 2560

(C) TÜBİTAK

doi:10.3906/mat-1905-110

\title{
Near-vector spaces determined by finite fields and their fibrations
}

Karin-Therese HOWELL*

Department of Mathematical Sciences, Faculty of Science, Stellenbosch University, Stellenbosch, South Africa

Received: 18.05.2019 • $\quad$ Accepted/Published Online: 28.08.2019 • Final Version: 28.09 .2019

\begin{abstract}
In this paper we study near-vector spaces constructed from copies of finite fields. We show that for these near-vector spaces regularity is equivalent to the quasikernel being the entire space. As a second focus, we study the fibrations of near-vector spaces. We define the pseudo-projective space of a near-vector space and prove that a special class of near-vector spaces, namely those constructed using finite fields, always has a fibration associated with them. We also give a formula for calculating the cardinality of the pseudo-projective space for this class of near-vector spaces.
\end{abstract}

Key words: Near-vector spaces, nearfields, near-vector space fibrations

\section{Introduction}

The near-vector spaces we study in this paper were first introduced by André [1] in 1974. Their subspaces and mappings were studied in [5] and their decomposition in [3]. Near-vector spaces constructed from finite fields were characterised in [6] and more recently in [11] the number of near-vector spaces constructed from finite fields were counted.

Karzel studied a near-vector space that satisfies the left distributive law and wrote about its fibered groups in $[7,8]$. These are also mentioned in Wahling [13]. André's near-vector spaces differ considerably as we will see. It is natural to wonder what the fibered groups of his near-vector spaces are. This will be the focus of this paper, with a special interest in constructions of near-vector spaces using copies of a finite field.

In Section 2 we give the preliminary material of near-vector spaces. In Section 3 we focus on the fibrations of near-vector spaces. We show that a special class of near-vector spaces always has a fibration associated with them and define the pseudo-projective space of a near-vector space. In Section 4 we focus specifically on nearvector spaces constructed from copies of finite fields. These are the closest to traditional vector spaces. We prove that for these constructions regularity is equivalent to the quasikernel being the entire space and show how the decomposition theorem of near-vector spaces decomposes the quasikernel. We prove that these near-vector spaces are fibered groups, or can be decomposed into fibered groups and we give a formula for the cardinality of the associated pseudoprojective space. Finally, we show when the fibers and maximal regular subspaces of a near-vector spaces will coincide.

\section{Preliminary material}

We begin with some definitions we will need.

*Correspondence: kthowell@sun.ac.za

2010 AMS Mathematics Subject Classification: 51J15; 16Y30 
HOWELL/Turk J Math

Definition 2.1 A (right) nearfield is a set F together with two binary operations + and $\cdot$ such that

1. $(F,+)$ is a group;

2. $(F \backslash\{0\}, \cdot)$ is a group;

3. $(a+b) \cdot c=a \cdot c+b \cdot c$ for all $a, b, c \in F$.

Left nearfields are defined analogously and satisfy the left distributive law. We will use right nearfields throughout this paper. We refer the reader to $[9,10]$ for more on nearfields.

In [1] the notion of a near-vector space was defined as

Definition 2.2 ([1], Definition 4.1, p.9) A near-vector space is a pair $(V, A)$ which satisfies the following conditions:

1. $(V,+)$ is a group and $A$ is a set of endomorphisms of $V$;

2. A contains the endomorphisms 0 , id and $-i d$;

3. $A^{*}=A \backslash\{0\}$ is a subgroup of the group $A u t(V)$;

4. If $x \alpha=x \beta$ with $x \in V$ and $\alpha, \beta \in A$, then $\alpha=\beta$ or $x=0$, i.e. A acts fixed point free on $V$;

5. The quasikernel $Q(V)$ of $V$, generates $V$ as a group. Here, $Q(V)=\{x \in V \mid \forall \alpha, \beta \in A, \exists \gamma \in$ A such that $x \alpha+x \beta=x \gamma\}$.

We will write $Q(V)^{*}$ for $Q(V) \backslash\{0\}$ throughout his paper. The dimension of the near-vector space, $\operatorname{dim}(V)$, is uniquely determined by the cardinality of an independent generating set for $Q(V)$, called a basis of $V$ (see [1]). In [1] it was proved that if the dimension of $V$ is greater than 1 and $Q(V)=V$, $V$ would be a vector space, but this is not true in general and we now have several counterexamples. In [12] it was proved that finite-dimensional near-vector spaces can be characterised in the following way:

Theorem 2.3 ([12], Theorem 3.4, p.301) Let $(G,+)$ be a group and let $A=D \cup\{0\}$, where $D$ is a fixed point free group of automorphism of $G$. Then $(G, A)$ is a finite-dimensional near-vector space if and only if there exist a finite number of near-fields $F_{1}, \ldots, F_{m}$, semigroup isomorphisms $\psi_{i}:(A, \circ) \rightarrow\left(F_{i}, \cdot\right)$, and an additive group isomorphism $\Phi: G \rightarrow F_{1} \oplus \ldots \oplus F_{m}$ such that if $\Phi(g)=\left(x_{1}, \ldots, x_{m}\right)$, then $\Phi(g \alpha)=\left(x_{1} \psi_{i}(\alpha), \ldots, x_{m} \psi_{m}(\alpha)\right)$ for all $g \in G, \alpha \in A$.

According to this theorem we can specify a finite dimensional near-vector space by taking $n$ copies of a nearfield $F$ for which there are semigroup isomorphisms $\psi_{i}:(F, \cdot) \rightarrow(F, \cdot), i \in\{1, \ldots, n\}$. We then take $V:=F^{n}, n$ a positive integer, as the additive group of the near-vector space and define

$$
\left(x_{1}, \ldots, x_{n}\right) \alpha:=\left(x_{1} \psi_{1}(\alpha), \ldots, x_{n} \psi_{n}(\alpha)\right)
$$

for all $\alpha \in F$ and $i \in\{1, \ldots, n\}$. This is the type of construction we will use throughout this paper. If we use $m$ near-fields in the construction, then the dimension of $V$ is $m$.

The concept of regularity is a central notion in the study of near-vector spaces. 
HOWELL/Turk J Math

Definition 2.4 ([1], Definition 4.11, p.306) A near-vector space is regular if any two vectors of $Q(V)^{*}$ are compatible, i.e. if for any two vectors $u$ and $v$ of $Q(V)^{*}$ there exists a $\lambda \in A \backslash\{0\}$ such that $u+v \lambda \in Q(V)$.

We also have a very important theorem below:

Theorem 2.5 ([1], Theorem 4.13, p.306) (The Decomposition Theorem) Every near-vector space $V$ is the direct sum of regular near vector spaces $V_{j}(j \in J)$ such that each $u \in Q(V)^{*}$ lies in precisely one direct summand $V_{j}$. The subspaces $V_{j}$ are maximal regular near-vector spaces.

We will not include the proof here, but we briefly outline the procedure described in the proof used to decompose a near-vector space into its maximal regular subspaces:

1. Start by partitioning $Q(V)^{*}$ into sets $Q_{j}(j \in J)$ of mutually pairwise compatible vectors.

2. Let $B \subseteq Q(V)^{*}$ be a basis of $V$ and let $B_{j}:=B \cap Q_{j}$.

3. Let $V_{j}:=\left\langle B_{j}\right\rangle$ be the subspace of $V$ generated by $B_{j}$, then each $V_{j}$ is a maximal regular subspace of $V$ and $V$ is the direct sum of the $V_{j}$.

By the Uniqueness Theorem ([1], Theorem 4.14 p.12), this decomposition is unique, and it is called the canonical decomposition of $V$. Thus, it is clear why André referred to regular near-vector spaces as the building blocks of near-vector space theory. Note that $V=Q(V)$ implies that $V$ is regular, but the converse is not true in general (See [5], Example 5.2, p.10). Also, if $V$ is regular, $Q(V)$ consists of pairwise compatible vectors, so it is the only partition and thus it is its own decomposition into maximal regular subspaces. We begin with a simple example.

Example 2.6 ([4], Example 3.2, p.59) Put $V:=\left(\mathbb{Z}_{5}\right)^{4}$ and $F=\mathbb{Z}_{5}$. Let $\alpha$ in $F$ act as an endomorphism on $V$ by defining

$$
\left(x_{1}, x_{2}, x_{3}, x_{4}\right) \alpha:=\left(x_{1} \alpha, x_{2} \alpha^{3}, x_{3} \alpha^{3}, x_{4} \alpha\right)
$$

Then $(V, F)$ is a near-vector space with basis

$$
B=\{(1,0,0,0),(0,1,0,0),(0,0,1,0),(0,0,0,1)\} .
$$

Hence, $V$ is a near-vector space of dimension four.

The quasikernel $Q(V)$ of $V$ consists of all those elements $u$ of $V$ such that for every $\alpha, \beta \in F$ there exists a $\gamma \in F$ for which $u \alpha+u \beta=u \gamma$. It is not difficult to check that

$$
Q(V)=\{(a, 0,0, d) \mid a, d \in F\} \cup\{(0, b, c, 0) \mid b, c \in F\} .
$$

By completing the steps of the decomposition theorem we obtain that the canonical decomposition of $V$ is given by $V=V_{1} \oplus V_{2}$, where

$$
V_{1}:=\left\langle B_{1}\right\rangle=\{(1,0,0,0) a+(0,0,0,1) d \mid a, d \in F\}=\{(a, 0,0, d) \mid a, d \in F\}
$$

and

$$
V_{2}:=\left\langle B_{2}\right\rangle=\{(0,1,0,0) b+(0,0,1,0) c \mid b, c \in F\}=\{(0, b, c, 0) \mid b, c \in F\}
$$


We end off with the following:

Definition 2.7 ([6], Definition 3.2., p. 57) We say that two near-vector spaces $\left(V_{1}, A_{1}\right)$ and $\left(V_{2}, A_{2}\right)$ are isomorphic (written $\left(V_{1}, A_{1}\right) \cong\left(V_{2}, A_{2}\right)$ ) if there are group isomorphisms $\theta:\left(V_{1},+\right) \rightarrow\left(V_{2},+\right)$ and $\eta:$ $\left(A_{1}^{*}, \cdot\right) \rightarrow\left(A_{2}^{*}, \cdot\right)$ such that $\theta(x \alpha)=\theta(x) \eta(\alpha)$ for all $x \in V_{1}$ and $\alpha \in A_{1}^{*}$.

We will write a near-vector space isomorphism as a pair $(\theta, \eta)$.

The following result was first proved by Mr S.P. Sanon.

Theorem 2.8 If the near-vector spaces $\left(V_{1}, A_{1}\right)$ and $\left(V_{2}, A_{2}\right)$ are isomorphic, say $(\theta, \eta)$ is the isomorphism, then $\theta\left(Q\left(V_{1}\right)\right)=Q\left(V_{2}\right)$.

Proof Let $v \in \theta\left(Q\left(V_{1}\right)\right)$, then $v=\theta\left(v^{\prime}\right)$ for some $v^{\prime} \in Q\left(V_{1}\right)$. If $\alpha, \beta \in A_{2}^{*}$, then since $\eta$ is an isomorphism, there exist $\alpha_{1}, \beta_{1} \in A_{1}^{*}$ such that $\eta\left(\alpha_{1}\right)=\alpha$ and $\eta\left(\beta_{1}\right)=\beta$. Thus,

$$
\begin{aligned}
v \alpha+v \beta & =v \eta\left(\alpha_{1}\right)+v \eta\left(\beta_{1}\right) \\
= & \theta\left(v^{\prime}\right) \eta\left(\alpha_{1}\right)+\theta\left(v^{\prime}\right) \eta\left(\beta_{1}\right) \\
= & \theta\left(v^{\prime} \alpha_{1}+v^{\prime} \beta_{1}\right) \\
= & \theta\left(v^{\prime} \gamma_{1}\right) \text { for some } \gamma_{1} \in A_{1}^{*}, \\
= & \theta\left(v^{\prime}\right) \eta(\gamma) \\
= & v \gamma \text { where } \gamma \in A_{2}^{*} .
\end{aligned}
$$

Thus, $v \in Q\left(V_{2}\right)$. A similar argument shows that $Q\left(V_{2}\right) \subseteq \theta\left(Q\left(V_{1}\right)\right)$ and so we have equality.

From this result, we can show that

Corollary 2.9 If the near-vector spaces $\left(V_{1}, A_{1}\right)$ and $\left(V_{2}, A_{2}\right)$ are isomorphic and $Q\left(V_{1}\right)=V_{1}$, then $Q\left(V_{2}\right)=$ $V_{2}$.

\section{Near-vector spaces and their fibrations}

We begin by recalling a few geometric definitions we will need:

Definition 3.1 A fibered group $(V,+, \mathcal{F})$, with identity 0 is a group $(V,+)$ with a fibration, i.e. a set $\mathcal{F}$ of subgroups of $V$ such that any element of $V$ different from 0 belongs to exactly one of such subgroups. The subgroups are called the fibers of $\mathcal{F}$.

Unlike in vector spaces, only a particular class of near-vector spaces has a natural fibration associated with them.

Theorem 3.2 Let $(V, A)$ be a near-vector space. Then $(V,+, \mathcal{F})$ is a fibered group where $\mathcal{F}=\left\{b A \mid b \in Q(V)^{*}\right\}$ if and only if $Q(V)=V$.

Proof Suppose that $(V,+, \mathcal{F})$ is a fibered group where $\mathcal{F}=\left\{b A \mid b \in Q(V)^{*}\right\}$. We already have that $Q(V) \subseteq V$. Now let $v \in V$, then since $(V,+, \mathcal{F})$ is a fibered group, there exists an $b^{\prime} \in Q(V)^{*}$ such that $v \in b^{\prime} A$, 
i.e. $v=b^{\prime} \lambda$ for some $\lambda \in A$. However, we know that the quasikernel is closed under scalar multiplication (refer to [1]), so $v \in Q(V)$. Thus, $Q(V)=V$. Conversely, suppose $Q(V)=V$. Since $0 \in(b A,+)$, it is nonempty. If $b \lambda_{1}, b \lambda_{2} \in b A$, then

$$
\begin{aligned}
b \lambda_{1}-b \lambda_{2} & =b \lambda_{1}+b\left(-\lambda_{2}\right) \\
& =b \gamma
\end{aligned}
$$

for some $\gamma \in A$ since $b \in Q(V)^{*}$. Thus, $(b A,+)$ is a subgroup of $(V,+)$. Since $(A, \circ)$ is a group of automorphisms of $(V,+)$ and $b A \subseteq V$, the scalar multiplication $(v, \lambda) \rightarrow v \lambda$ defines an action on $V$ so that $V=\bigcup_{b \in Q(V)^{*}} b A$.

We note that the fibers in the theorem above are just the orbits of the action of $A$ on $V^{*}$. Thus, we are investigating when the orbits of the action of $A$ on $V^{*}$ will be subgroups of $(V,+)$.

A natural question to ask is given a fibration, can we associate a near-vector space with it?

Lemma 3.3 Let $(V,+, \mathcal{F})$ be a fibered group where $\mathcal{F}=\left\{F_{i}\right\}$ and the $F_{i}$ are isomorphic nearfields for $i \in\{1, \ldots, n\}$. Then $\left(V^{\prime}, F_{j}\right)$ is a near-vector space for each $j \in\{1, \ldots, n\}$ where $V^{\prime}=F_{1} \oplus \ldots \oplus F_{n}$.

Proof Put $V^{\prime}=F_{1} \oplus \ldots \oplus F_{n}$ and pick some $j \in\{1, \ldots, n\}$. Now consider for each $i \in\{1, \ldots, n\}$, $\psi_{i}=\left(F_{j}, \cdot\right) \rightarrow\left(F_{i}, \cdot\right)$, then we define the scalar multiplication for all $\alpha \in F_{j}$ as

$$
\left(x_{1}, \ldots, x_{n}\right) \alpha=\left(x_{1} \psi_{1}(\alpha), \ldots, x_{n} \psi_{n}(\alpha)\right)
$$

and by van der Walt's Theorem $2.3,\left(V^{\prime}, F_{j}\right)$ is a near-vector space.

We now define a new relation on $V$ :

Definition 3.4 Let $(V, A)$ be a near-vector space. We define a relation on $V$ such that for $u, v \in V, u \asymp v$ iff $v=u \lambda$ for some $\lambda \in A^{*}=A \backslash\{0\}$.

Lemma 3.5 Let $(V, A)$ be a near-vector space. The relation $\asymp$ defined above is an equivalence relation on $V$.

We also define, as for vector spaces,

Definition 3.6 Let $(V, A)$ be a near-vector space. Then the pseudo-projective space $P(V)$ induced by $V$ is the set of equivalence classes in $V \backslash\{0\}$ under the equivalence relation defined by $\asymp$.

We refer to $P(V)$ as the pseudo-projective space associated with a near-vector space since it is not clear yet under which conditions $P(V)$ will be a projective space. It turns out that the the fibers of Theorem 3.2 are the nonzero equivalence classes of the relation $\asymp$.

Lemma 3.7 Let $(V, A)$ be a near-vector space with $Q(V)=V$. Then the nonzero equivalence classes of the relation $\asymp$ are exactly the fibers $b A^{*}$ for $b \in Q(V)^{*}$.

Then we immediately have that

Lemma 3.8 Let $(V, A)$ be a near-vector space with $Q(V)=V$, then $P(V)=\left\{b A^{*} \mid b \in Q(V)^{*}\right\}$. 
HOWELL/Turk J Math

We also have:

Proposition 3.9 Let $(V, A)$ be a near-vector space with $Q(V)=V$ and for all $\lambda \in A^{*}$ define the map $\lambda_{o}: V \backslash\{0\} \rightarrow V \backslash\{0\}$ by $x \rightarrow x \lambda$. Then $\lambda_{0}$ maps each fiber bA* for $b \in Q(V)^{*}$ to itself.

Next we prove that isomorphisms preserve fibrations,

Proposition 3.10 Let $(V, A)$ and $\left(V^{\prime}, A^{\prime}\right)$ be near-vector spaces such that $(V,+, \mathcal{F})$ is a fibered group, where $\mathcal{F}=\left\{b A \mid b \in Q(V)^{*}\right\}$. Let $(\theta, \eta)$ be an isomorphism between $V$ and $V^{\prime}$. Then $\theta(\mathcal{F})=\left\{\theta(b A) \mid b \in Q(V)^{*}\right\}$ is a fibration of $V^{\prime}$.

Proof Since $(V,+, \mathcal{F})$ is a fibered group by Theorem 3.2, $Q(V)=V$ so by Corollary $2.9, Q\left(V^{\prime}\right)=V^{\prime}$ and for each $b \in Q(V)^{*}$,

$$
\begin{aligned}
\theta(b A) & =\theta(b) \eta(A) \\
& =b^{\prime} A^{\prime} \text { for some } b^{\prime} \in Q\left(V^{\prime}\right)^{*} \text { by Theorem } 2.8 .
\end{aligned}
$$

Now by Theorem 3.2, $\theta(\mathcal{F})=\left\{\theta(b A) \mid b \in Q(V)^{*}\right\}$ is a fibration of $V^{\prime}$.

It is natural to ask what the connection between the compatibility relation and this new relation is. We can show that:

Lemma 3.11 Let $(V, A)$ be a near-vector space and $u, v \in V$, with $u \asymp v$, then $u \sim v$.

Proof Let $u, v \in V$, with $u \asymp v$, then $v=u \lambda$ for some $\lambda \in F^{*}$. Moreover, by a known property of the quasikernel ([1]) $u+u \lambda \in Q(V)$, so $u \sim v$.

In the next example we show that $u \sim v$ does not in general imply that $u \asymp v$.

Example 3.12 Referring back to Example 2.6, we note that $(1,0,0,1) \sim(3,0,0,2)$ since $(1,0,0,1)+(3,0,0,2)=$ $(4,0,0,3) \in Q(V)^{*}$, but clearly $(1,0,0,1) \nsucc(3,0,0,2)$.

\section{Finite dimensional near-vector spaces constructed using finite fields}

In this section we focus on constructions of near-vector spaces using Theorem 2.3, beginning with a finite field. Thus, let $F:=G F\left(p^{r}\right)$ be the Galois field of $p^{r}$ elements where $p$ is a prime and $r$ a positive integer. We put $V:=F^{n}, n$ a positive integer and use semigroup isomorphisms $\psi_{i}:(F, \cdot) \rightarrow(F, \cdot), i \in\{1, \ldots, n\}$ to define the scalar multiplication as

$$
\left(x_{1}, \ldots, x_{n}\right) \alpha:=\left(x_{1} \psi_{1}(\alpha), \ldots, x_{n} \psi_{n}(\alpha)\right)
$$

for all $\left(x_{1}, \ldots, x_{n}\right) \in V$ and $\alpha \in F$, where the $\psi_{i}^{\prime} s$ can be equal.

The construction and counting of such near-vector spaces were studied in [6] and subsequently in [11].

The following theorem was recently proved. It characterises regularity for these near-vector spaces.

Lemma 4.1 ([2], Lemma 5.8, p.11) Let $F=G F\left(p^{r}\right)$ and $V=F^{n}$ be a near-vector space with scalar multiplication defined for all $\alpha \in F$ by

$$
\left(x_{1}, \ldots, x_{n}\right) \alpha:=\left(x_{1} \psi_{1}(\alpha), \ldots, x_{n} \psi_{n}(\alpha)\right)
$$


where the $\psi_{i}^{\prime} s$ are automorphisms of $(F, \cdot)$. Then $V$ is regular if and only if for all $i, j \in I$ and $\alpha \in G F\left(p^{r}\right)$, $\psi_{i}(\alpha)=\psi_{j}\left(\alpha^{p^{l}}\right)$, for some $l \in\{0,1, \ldots, r-1\}$.

In fact, in [2] using this theorem it is shown that if $A_{1}, A_{2}, \ldots, A_{k}$ is a partition of the set $I=\{1, \ldots, n\}$, where

$$
A_{i}:=\left\{j \in I \mid \psi_{i}(\alpha)=\psi_{j}\left(\alpha^{p^{l}}\right) \text { for some } l \in\{0,1, \ldots, r-1\}\right\}
$$

then

Lemma 4.2 ([2], Lemma 5.11, p.14) For the near-vector space defined above we have that:

1. $Q(V)=\bigcup_{t=1}^{k} \mathcal{V}_{t}$ where

$$
\mathcal{V}_{t}=\left\{\left(0,0, \ldots, a_{1}, 0, a_{2}, 0, \ldots, a_{s}, 0\right) \mid a_{i} \text { is in position } \ell \text { with } \ell \in A_{t}, \text { for } t \in K\right\}
$$

where $K:=\{1, \ldots, k\}$.

2. Each of the $\mathcal{V}_{t}$ is a regular subspace of $V$.

3. $V=\mathcal{V}_{1} \oplus \mathcal{V}_{2} \oplus \cdots \oplus \mathcal{V}_{k}$ is the canonical decomposition of $V$.

This corrects an error in [5] where it was stated that the partition of the set $I=\{1, \ldots, n\}$, was given by

$$
A_{i}:=\left\{j \in I \mid \psi_{i}(\alpha)=\psi_{j}(\alpha)\right\}
$$

(refer to page 8 in [5]). This is in fact the partition for near-vector spaces constructed with copies of $\mathbb{Z}_{p}$ for $p$ a prime, since in this case $r=1$ in Lemma 4.1 .

We have already mentioned that in general $V$ being regular does not imply that $Q(V)=V$, but for the constructions using copies of finite fields this is the case. Although the proof is short, this is an important result in near-vector space theory.

Theorem 4.3 Let $F=G F\left(p^{r}\right)$ and $V=F^{n}$ be a near-vector space with scalar multiplication defined for all $\alpha \in F$ by

$$
\left(x_{1}, \ldots, x_{n}\right) \alpha:=\left(x_{1} \psi_{1}(\alpha), \ldots, x_{n} \psi_{n}(\alpha)\right),
$$

where the $\psi_{i}^{\prime} s$ are automorphisms of $(F, \cdot)$. Then the following are equivalent

1. $Q(V)=V$;

2. $V$ is regular;

3. for all $i, j \in\{1, \ldots, n\}$ and $\alpha \in G F\left(p^{r}\right), \psi_{i}(\alpha)=\psi_{j}\left(\alpha^{p^{l}}\right)$, for some $l \in\{0,1, \ldots, r-1\}$.

Proof By Lemma 4.1, 2. and 3. are equivalent. Suppose that $Q(V)=V$, then $V$ is regular, so 3. follows from Lemma 4.1. Now suppose that for all $i, j \in\{1, \ldots, n\}$ and $\alpha \in G F\left(p^{r}\right), \psi_{i}(\alpha)=\psi_{j}\left(\alpha^{p^{l}}\right)$, for some $l \in\{0,1, \ldots, r-1\}$. Then the partition of $I$ described above in Lemma 4.2 has one cell, $A_{1}=I$, so by Lemma 4.2, $Q(V)=\mathcal{V}$, where $\mathcal{V}=\left\{\left(a_{1}, a_{2}, \ldots, a_{n}\right) \mid a_{i} \in F\right\}$, thus $Q(V)=V$.

As an example, consider 
Example 4.4 Let $F=G F\left(3^{2}\right), V=F^{2}$ and for all $\alpha \in F,\left(x_{1}, x_{2}\right) \in V$, define $x \alpha=\left(x_{1} \alpha, x_{2} \alpha^{3}\right)$. If we let $\psi_{1}(\alpha)=\alpha$ and $\psi_{2}(\alpha)=\alpha^{3}$, then

$$
\begin{aligned}
\psi_{2}\left(\alpha^{3}\right) & =\alpha^{9} \\
& =\alpha \text { since } \alpha^{9} \cong \alpha(\bmod 8) \\
& =\psi_{1}(\alpha) .
\end{aligned}
$$

Thus, by Theorem 4.3, $Q(V)=V$ and $V$ is regular.

Finally, we can show that the decomposition theorem also decomposes the quasikernel.

Theorem 4.5 Let $F=G F\left(p^{r}\right)$ and $V=F^{n}$ be a near-vector space with scalar multiplication defined for all $\alpha \in F$ by

$$
\left(x_{1}, \ldots, x_{n}\right) \alpha:=\left(x_{1} \psi_{1}(\alpha), \ldots, x_{n} \psi_{n}(\alpha)\right)
$$

where the $\psi_{i}^{\prime}$ s are automorphisms of $(F, \cdot)$. If $Q(V) \neq V$ and $V=V_{1} \oplus \cdots \oplus V_{k}$ is the canonical decomposition of $V$, then $Q(V)=Q_{1} \cup \cdots \cup Q_{k}$ where $Q_{i}=V_{i}$ for each $i \in\{1, \ldots, k\}$.

Proof By Lemma 4.2,

$$
Q(V)=\bigcup_{i=1}^{k} Q_{i}
$$

where $Q_{j}=\left\{\left(a_{1}, 0, \ldots, a_{i}, 0\right) \mid a_{i}\right.$ in position $i$ with $i \in A_{j}$ for $\left.j \in\{1, \ldots, k\}\right\}$; moreover, this is the partition of $Q(V) \backslash\{0\}$ into sets $Q_{1}=Q_{1} \backslash\{0\}, \ldots, Q_{k}=Q_{k} \backslash\{0\}$ of mutually compatible vectors. If we now intersect each of these with the canonical basis $B$ of $V$, and let $B_{j}:=B \cap Q_{j}$ for $i \in\{1, \ldots, k\}$ and consider $V_{j}:=$ $\left\langle B_{j}\right\rangle$, then all finite sums and scalar multiples of elements of $B_{j}$ again gives rise to elements of the form of $Q_{j}$, so that for each $j \in\{1, \ldots, k\}, V_{j}=Q_{j}$.

Example 4.6 Referring back to Example 2.6, with $V:=\left(\mathbb{Z}_{5}\right)^{4}$ and $F=\mathbb{Z}_{5}$. Let $\alpha$ in $F$ act as an endomorphism on $V$ by defining $\left(x_{1}, x_{2}, x_{3}, x_{4}\right) \alpha:=\left(x_{1} \alpha, x_{2} \alpha^{3}, x_{3} \alpha^{3}, x_{4} \alpha\right)$.

$$
Q(V)=(\{(a, 0,0, d) \mid a, d \in F\} \cup\{(0, b, c, 0) \mid b, c \in F\})
$$

whilst $V=V_{1} \oplus V_{2}$ with $V_{1}=\{(a, 0,0, d) \mid a, d \in F\}$ and $V_{2}=\{(0, b, c, 0) \mid b, c \in F\}$.

We now show that in the case where we begin the construction with a finite field, we can always associate a fibered group with it.

Theorem 4.7 Any near-vector space of the form $V=F^{n}$ where $F=G F\left(p^{r}\right)$ and scalar multiplication defined for all $\alpha \in F$ by

$$
\left(x_{1}, \ldots, x_{n}\right) \alpha:=\left(x_{1} \psi_{1}(\alpha), \ldots, x_{n} \psi_{n}(\alpha)\right)
$$

where the $\psi_{i}^{\prime}$ s are automorphisms of $(F, \cdot)$, is a fibered group, or can be decomposed into fibered groups. 
HOWELL/Turk J Math

Proof There are two cases to consider:

Case 1: $V$ is regular

Then by Theorem 4.3, $Q(V)=V$, so by Theorem 3.2, $(V,+, \mathcal{F})$ is a fibered group where $\mathcal{F}=\left\{a F \mid a \in Q(V)^{*}\right\}$.

Case 2: $V$ is not regular

Suppose that $V=V_{1} \oplus V_{2} \oplus \cdots \oplus V_{r}$, is the canonical decomposition of $V$ into maximal regular subspaces. Then by Theorem 4.3, for $i \in\{1,2, \ldots, k\}$, each $V_{i}$ has $Q\left(V_{i}\right)=V_{i}$. Now by Theorem 3.2 each $V_{i}, i \in\{1,2, \ldots, k\}$, is a fibered group, $\left(V_{i}, \mathcal{F}_{i}\right)$, where $\mathcal{F}_{i}=\left\{a F \mid a \in Q_{i}{ }^{*}\right\}=\left\{a F \mid a \in V_{i}^{*}\right\}$ for $i \in\{1, \ldots, k\}$.

Taking this back to the orbits of the action of $A$ on $V^{*}$, we see that for these constructions, either we begin with the orbits being subgroups of $(V,+)$ or we can decompose so that they will be.

Here are two examples of the two cases:

Example 4.8 Returning to Example 4.4, $(V,+, \mathcal{F})$ is a fibered group where $\mathcal{F}=\left\{\left(x_{1}, x_{2}\right) F \mid\left(x_{1}, x_{2}\right) \in V^{*}\right\}$.

Example 4.9 Returning to Example 2.6, if $V:=\left(\mathbb{Z}_{5}\right)^{4}$ and $F=\mathbb{Z}_{5}$ and $\alpha$ in $F$ acts as an endomorphism on $V$ by $\left(x_{1}, x_{2}, x_{3}, x_{4}\right) \alpha:=\left(x_{1} \alpha, x_{2} \alpha^{3}, x_{3} \alpha^{3}, x_{4} \alpha\right)$, then as we saw

$$
Q(V)=\{(a, 0,0, d) \mid a, d \in F\} \cup\{(0, b, c, 0) \mid b, c \in F\}
$$

By the decomposition theorem, $V_{1}$ and $V_{2}$ are maximal regular near-vector spaces and the canonical decomposition of $V$ is given by $V=V_{1} \oplus V_{2}$ where,

$$
V_{1}:=\{(a, 0,0, d) \mid a, d \in F\}
$$

and

$$
V_{2}:=\{(0, b, c, 0) \mid b, c \in F\}
$$

Thus, as we saw by the previous theorem, $\left(V_{i}, \mathcal{F}_{i}\right)$ is a fibered group for $i \in\{1,2\}$, where

$$
\mathcal{F}_{1}=\{\{(0,0,0,1) F\},\{(1,0,0,0) F\},\{(1,0,0,1) F\},\{(1,0,0,2) F\},\{(0,0,0,3) F\},\{(1,0,0,4) F\}\}
$$

and

$$
\mathcal{F}_{2}=\{\{(0,1,0,0) F\},\{(0,1,1,0) F\},\{(0,0,1,0) F\},\{(0,1,2,0) F\},\{(0,1,3,0) F\},\{(0,1,4,0) F\}\} .
$$

Returning now to the pseudo-projective space of a near-vector space constructed from a finite field, we begin by remarking that if we chose all the $\psi_{i}$ 's to be the identity, we get the scalar multiplication

$$
\left(x_{1}, \ldots, x_{n}\right) \alpha:=\left(x_{1} \psi_{1}(\alpha), \ldots, x_{n} \psi_{n}(\alpha)\right)
$$

for all $\left(x_{1}, \ldots, x_{n}\right) \in V, \alpha \in F$ and $(V, F)$ is a vector space. It is well known that in this case, if $V$ has dimension 3, then $P(V)$ is a projective space. In general $P(V)$ has order $\frac{p^{r n}-1}{p^{r}-1}$ for vector spaces. In fact, for all regular near-vector spaces in our construction, we have

Lemma 4.10 Let $F=G F\left(p^{r}\right)$ and $V=F^{n}$ be a near-vector space with scalar multiplication defined for all $\alpha \in F$ by

$$
\left(x_{1}, \ldots, x_{n}\right) \alpha:=\left(x_{1} \psi_{1}(\alpha), \ldots, x_{n} \psi_{n}(\alpha)\right)
$$

where the $\psi_{i}^{\prime}$ s are automorphisms of $(F, \cdot)$. If $V$ is regular, $|P(V)|=\frac{p^{r n}-1}{p^{r}-1}$. 
HOWELL/Turk J Math

Proof Since $V$ is regular, $Q(V)=V$, so by Theorem 3.2, $(V,+, \mathcal{F})$ is a fibered group where $\mathcal{F}=\left\{a F \mid a \in V^{*}\right\}$. Now since the fibers are the equivalence classes of $\asymp$, and $V$ has $p^{r n}-1$ nonzero elements and each fiber has $p^{r}-1$ nonzero elements, we get that $P(V)=\frac{p^{r n}-1}{p^{r}-1}$.

In the case where $V$ is not regular, we have that

Lemma 4.11 Let $F=G F\left(p^{r}\right)$ and $V=F^{n}$ be a near-vector space with scalar multiplication defined for all $\alpha \in F$ by

$$
\left(x_{1}, \ldots, x_{n}\right) \alpha:=\left(x_{1} \psi_{1}(\alpha), \ldots, x_{n} \psi_{n}(\alpha)\right),
$$

where the $\psi_{i}^{\prime}$ s are automorphisms of $(F, \cdot)$. If $V$ is not regular, $|P(V)|=\sum_{i=1}^{k} \frac{p^{r n_{i}}-1}{p^{r}-1}$.

Proof Suppose that $V=V_{1} \oplus V_{2} \oplus \cdots \oplus V_{k}$, is the canonical decomposition of $V$ and $n_{1}, \ldots, n_{k}$ are the dimensions of $V_{1}, \ldots, V_{k}$. Then by Theorem 4.7, the fibers are of the form $\mathcal{F}_{i}=\left\{a F \mid a \in V_{i}^{*}\right\}$ and since the total number of nonzero elements in $V_{i}$ is $p^{n_{i}}-1$, for $i \in\{1, \ldots, k\}$ and each fibration has $p^{r}-1$ nonzero elements, we have that $|P(V)|=\sum_{i=1}^{k} \frac{p^{r n_{i}}-1}{p^{r}-1}$.

Example 4.12 Returning to Examples 4.8 and 4.9, using our formula we get that $|P(V)|=\frac{80}{8}=10$ and $|P(V)|=\frac{24}{4}+\frac{24}{4}=12$, respectively.

Finally, we might wonder when, for the construction under consideration, the fibers and maximal subspaces coincide.

Theorem 4.13 Let $F=G F\left(p^{r}\right)$ and $V=F^{n}$ be a near-vector space with scalar multiplication defined for all $\alpha \in F$ by

$$
\left(x_{1}, \ldots, x_{n}\right) \alpha:=\left(x_{1} \psi_{1}(\alpha), \ldots, x_{n} \psi_{n}(\alpha)\right),
$$

where the $\psi_{i}^{\prime} s$ are automorphisms of $(F, \cdot)$ and for all $i, j \in I$ and $\alpha \in G F\left(p^{r}\right), \psi_{i}(\alpha) \neq \psi_{j}\left(\alpha^{p^{l}}\right)$, then $(V, \mathcal{F})$ is a fibration where the fibers are the maximal regular subspaces in the canonical decomposition of $V$.

Proof By Lemma 4.1, $V$ is not regular, so by Theorem 4.7 it can be decomposed into fibered groups. As discussed in Section 3, we begin with the partition of the set $I=\{1, \ldots, n\}$, where $A_{i}:=\left\{j \in I \mid \psi_{i}(\alpha)=\right.$ $\psi_{j}\left(\alpha^{p^{l}}\right)$ for some $\left.l \in\{0,1, \ldots, r-1\}\right\}$, so here we will have $A_{1}, A_{2}, \ldots, A_{n}$ with $A_{i}=\{i\}$ for $i \in I$. By Lemma $4.2(1)$, in general for this construction, we have that $Q(V)=\bigcup_{t=1}^{n} \mathcal{V}_{t}$ where

$$
\mathcal{V}_{t}=\left\{\left(0,0, \ldots, a_{1}, 0, a_{2}, 0, \ldots, a_{s}, 0\right) \mid a_{i} \text { is in position } \ell \text { with } \ell \in A_{t}, \text { for } t \in K\right\}
$$

where $K:=\{1, \ldots, k\}$. In this particular case,

$$
\mathcal{V}_{t}=\left\{\left(0,0, \ldots, a_{t}, 0,0,0, \ldots, 0,0\right) \mid a_{t} \text { is in position } t \text { for } t \in\{1, \ldots, n\}\right\} .
$$

If we now take an arbitrary element of $\mathcal{V}_{t}$, say $\left(0,0, \ldots, a_{t}, 0,0,0, \ldots, 0,0\right)$ then it is not difficult to see that

$$
\left(0,0, \ldots, a_{t}, 0,0,0, \ldots, 0,0\right) F=\left\{\left(0,0, \ldots, s_{t}, 0,0,0, \ldots, 0,0\right) \mid s_{t} \in F\right\}=\mathcal{V}_{t},
$$


HOWELL/Turk J Math

and by Lemma 4.2(2), these are exactly the maximal regular subspaces; thus, the maximal regular subspaces and fibers coincide.

Corollary 4.14 For the near-vector space $(V, F)$ of Theorem 4.13, $|P(V)|=n$.

We close with an example,

Example 4.15 Consider the near-vector space $(V, F)$, where $V:=\left(\mathbb{Z}_{5}\right)^{2}$ and $F=\mathbb{Z}_{5}$ and $\alpha$ in $F$ acts as an endomorphism on $V$ by $\left(x_{1}, x_{2}\right) \alpha:=\left(x_{1} \alpha, x_{2} \alpha^{3}\right)$, then

$$
Q(V)=\{(a, 0) \mid a \in F\} \cup\{(0, b) \mid b \in F\}
$$

By the decomposition theorem, the canonical decomposition of $V$ is given by $V=V_{1} \oplus V_{2}$ where,

$$
V_{1}:=\{(a, 0) \mid a \in F\}
$$

and

$$
V_{2}:=\{(0, b) \mid b \in F\}
$$

Thus, as we saw by the previous theorem, $V_{1}$ and $V_{2}$ are fibered groups and $|P(V)|=2$.

\section{Questions for future work}

In the future I hope to study the geometric structure of the pseudoprojective space.

\section{Acknowledgments}

The author is grateful for funding by the National Research Fund (South Africa) (Grant number: 96056) and helpful conversations with Mr S.P. Sanon and Prof. P. Cara. This paper is dedicated to the memory of Duane Mulder (28/08/1980-03/06/1993), a sunshine kid, gone too soon.

\section{References}

[1] André J. Lineare Algebra über Fastkörpern. Mathematische Zeitschrift 1974; 136: 295-313 (in German).

[2] Chistyakov D, Howell K-T, Sanon SP. On representation theory and near-vector spaces. Linear Multilinear Algebra 2019; 67 (7): 1495-1510. doi: 10.1080/03081087.2018.1459449

[3] Dorfling S, Howell K-T, Sanon SP. The decomposition of finite dimensional Near-vector spaces. Communications in Algebra 2018; 46 (7): 13033-3046. doi: 10.1080/00927872.2017.1404083

[4] Howell K-T. Contributions to the Theory of Near-Vector Spaces. PhD, University of the Free State, Bloemfontein, South Africa, 2008.

[5] Howell K-T. On subspaces and mappings of Near-vector spaces. Communications in Algebra 2015; 43 (6): $2524-2540$. doi: $10.1080 / 00927872.2014 .900689$

[6] Howell K-T, Meyer JH. Near-vector spaces determined by finite fields. Journal of Algebra 2010; 398: 55-62. doi: 10.1016/j.jalgebra.2013.09.019

[7] Karzel H. Fastvektorräume, unvollständige Fastkörper und ihre abgeleiteten Strukturen. In: Mitteilungen des Mathematischen Seminars der Universitaet Giessen; Giessen, Germany; 1984. pp.127 -139 (in German). 
HOWELL/Turk J Math

[8] Karzel H, Kist G. Determination of all Near Vector Spaces with Projective and Affine Fibrations. Journal of Geometry 1984; 23: 124-126.

[9] Meldrum JDP. Near-rings and Their Links with Groups. Newyork, NY, USA: Advanced Publishing Program, 1985.

[10] Pilz G. Near-rings: The Theory and its Applications, Revised Edition. New York, NY, USA: North Holland, 1983.

[11] Rodtes K, Chomjun W. On the number of near-vector spaces determined by finite fields. Journal of Algebra 2017; 492: 90-101. doi: 10.1016/j.jalgebra.2017.08.024

[12] van der Walt APJ. Matrix near-rings contained in 2-primitive near-rings with minimal subgroups. Journal of Algebra 1992; 148: 296-304.

[13] Wahling H. Theorie der Fastkörper. Thales Monographs, 1. Essen, DE: Thales-Verlag, 1987 (in German). 